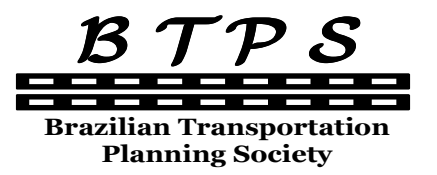

Planning Society
Journal of Transport Literature

Vol. 6, n. 4, pp. 178-189, Oct 2012

Reviews \& Essays
JTL | RELIT

www.transport-literature.org ISSN 2238-1031

\title{
Capacidade do lado aéreo dos aeroportos brasileiros
}

[Airside capacity of Brazilian airports]

\author{
Cláudio Jorge Pinto Alves*, Rafael Fraga \\ Instituto Tecnológico de Aeronáutica (ITA), Brazil, ISA Software LLC, USA
}

Submitted 20 May 2011; received in revised form 11 Jul 2011; accepted 26 Jan 2012

\begin{abstract}
Resumo
0 crescimento da demanda pelo transporte aéreo tem exigido ações para adequação da capacidade instalada nos aeroportos. Normalmente tais obras requerem vultosos recursos e trazem preocupações socioambientais. Uma ampliação em qualquer setor do aeroporto necessita uma visão sistêmica para garantir que a obra vai efetivamente propiciar ganhos ao aeroporto como um todo e não simplesmente se transferir o gargalo dentro do sistema. Neste artigo são mostrados os resultados dos cálculos das capacidades de dois subsistemas do lado aéreo dos vinte principais aeroportos brasileiros. Apenas em quatro aeroportos se mostra a ocorrência de um balanceamento entre as suas capacidades. Nos demais, um ou outro subsistema se caracteriza como limitante. Para esses casos, a ampliação da capacidade do aeroporto somente se viabiliza se as ações se voltarem para o subsistema limitado.
\end{abstract}

Palavras-Chave: capacidade aeroportuária; lado aéreo; pista; pátio; diagnóstico.

\begin{abstract}
In order to satisfy the continually growing demand for air transportation, further improvements has been demanded to increase airports' capacity. Usually, such effort requires significant resources, generating social and environmental concerns. When improving any airport facility, several systemic actions are required in order to ensure that such effort will actually be able to provide real benefits to the airport (as a whole), instead of just transferring the bottleneck through the system. In the present study, the operational capacities of two airside's subsystems are presented, showing those metrics for the top twenty Brazilian airports. As a result, only four of them indicate good balance between subsystems capacities, therefore, all the others are showing at least one subsystem that can be characterized as a bottleneck. For those cases, the expansion of the airport capacity is only possible through actions capable to solve capacity problems at the limited subsystem.
\end{abstract}

Key words: airport capacity; airside; runway; apron; diagnosis.

*Corresponding Author. Email: claudioj@ita.br.

\section{Recommended Citation}

Alves, C. J. P. and Fraga, R. (2012) Capacidade do lado aéreo dos aeroportos brasileiros. Journal of Transport Literature, vol. 6, n. 4, pp. 178-189.

- JTL/RELIT is a fully electronic, peer-reviewed, open access, international journal focused on emerging transport markets and published by BPTS - Brazilian Transport Planning Society. Website www.transport-literature.org. ISSN 2238-1031.

This paper is downloadable at www.transport-literature.org/open-access. 


\section{Introdução}

De 2009 a 2010, a taxa de crescimento de passageiros transportados pelo modal aéreo no país cresceu acima dos 20\% (INFRAERO, 2011). Atribui-se como causas, dentre outras: a melhoria da economia como um todo (PIB de 4,7\% ao ano no período de 2003 a 2008) e a inclusão dos passageiros da classe B e C como entrantes nesse mercado (McKinsey, 2010). No entanto, os investimentos em infraestrutura não acompanharam esse mesmo ritmo e se constatou a degradação acentuada do nível de serviço oferecido nos aeroportos brasileiros.

Desde 2006, independentes da realização da Copa do Mundo de Futebol em 2014 e dos Jogos Olímpicos de 2016 no Rio de Janeiro, estudos mostravam a situação preocupante em que se encontravam os principais aeroportos brasileiros (Carvalho, 2006).

Nenhuma obra de vulto foi completada ou iniciada de forma a mitigar os problemas de congestionamento e saturação desses aeroportos. Acidentes aéreos espetaculares desencadearam crises: o choque das aeronaves sobre o espaço aéreo do Mato Grosso pôs em cheque o controle do tráfego aéreo no Brasil e o overrun ${ }^{1}$ do A320 em Congonhas chamou a atenção das condições de segurança nos aeroportos. Autoridades do setor foram substituídas. Medidas anunciadas, mas, efetivamente, não se obteve a ampliação das capacidades, pelo contrário, em favor da segurança foram reduzidas as capacidades de algumas pistas de pouso.

As críticas provenientes dos mais diversos segmentos da sociedade (e mesmo de entidades estrangeiras) têm pressionado o Governo Federal a tomar providências (na infraestrutura do País) que garantam a realização dos megaeventos em condições adequadas. A expansão da infraestrutura é um processo complexo e tecnicamente consumidor de tempo, além do alto custo envolvido, tanto financeiro como socioambiental. A própria participação da Iniciativa Privada depende de marcos regulatórios ainda não bem estabelecidos pelo Poder Público.

Diante dessa situação, qualquer medida que possa tornar mais eficiente a operação das instalações já existentes passa a ganhar relevância. O aeroporto é um sistema composto por vários subsistemas: pistas, pátios, terminais, acessos etc. Ainda que medidas que melhorem quaisquer um desses elos sejam importantes, é fundamental que o sistema seja analisado pelo

\footnotetext{
${ }^{1}$ excursão de pista na qual a aeronave ultrapassa a cabeceira oposta ao toque (FSF, 2009).
} 
seu conjunto. A capacidade de um aeroporto depende da capacidade de cada um dos seus subsistemas. Capacidade, segundo Gualda (1995), pode ser entendida como o nível máximo de demanda que pode ser imposto a um subsistema, em um dado período de tempo, sem violar nenhum dos critérios de níveis de serviço estipulados.

Na prática, a avaliação da capacidade de um complexo aeroportuário ainda é motivo de muita discussão técnica. Em maio de 2011 a INFRAERO, empresa que administra os mais movimentados aeroportos brasileiros, anunciou uma mudança de referenciais de capacidade o que possibilitou anunciar que vários aeroportos saturados passassem a condição de estarem com um nível satisfatório de operação. Enfim, conceitos como nível de serviço e hora-pico (ou hora de projeto) não apresentam na literatura uma definição única e consagrada, permitindo variações que dificultam comparações quantitativas.

Os subsistemas do lado aéreo de um aeroporto têm como variações de nível de serviço, dentre outros, a separação a ser mantida entre aeronaves ou operações e os tempos de espera aceitáveis (atrasos) para as operações. Os primeiros são limitados inferiormente, por motivo de segurança, pela ICAO (Organização da Aviação Civil Internacional).

Esse trabalho analisa a capacidade do Lado Aéreo de aeroportos brasileiros e verifica o balanceamento de dois dos seus subsistemas: pistas e pátios de estacionamento de aeronaves. A abordagem se baseia em diagnósticos sobre 20 dos maiores aeroportos brasileiros. Esse conjunto atendeu em 2010 a cerca de $70 \%$ do movimento de aeronaves e a $90 \%$ do movimento de passageiros no Brasil.

Além de avaliar as capacidades dos subsistemas pistas e pátios de estacionamento das aeronaves, revela-se, também, para cada subsistema, as medidas que podem ser tomadas para ampliação de sua capacidade operacional. Finalmente procura-se enfatizar a importância do balanceamento entre os subsistemas para a obtenção de efetivos ganhos na capacidade de um aeroporto. Na Figura 1, na imagem do lado aéreo do Aeroporto Internacional de São Paulo / Guarulhos (GRU) está destacado (circulado em vermelho) o subsistema pátios do lado aéreo e se registra em amarelo a designação de cada via do subsistema pistas. 


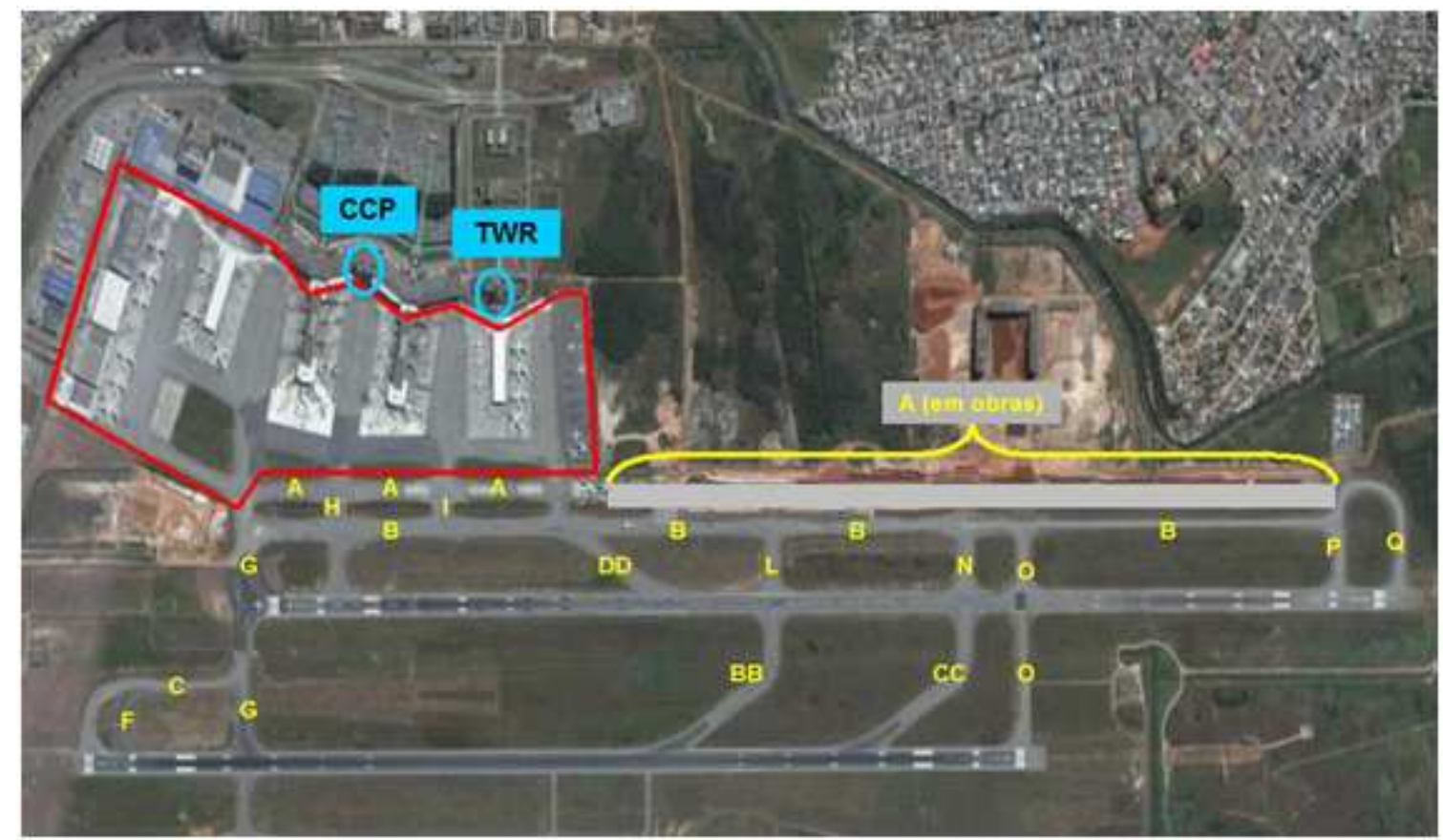

Figura 1 - Lado aéreo de GRU (Fonte: Google Earth)

O presente trabalho analisa na Seção 2 a capacidade do subsistema pistas referentes aos 20 aeroportos em estudo. Na Seção 3, a capacidade do subsistema pátio desses mesmos aeroportos. Na Seção 4 se identifica o subsistema limitante no lado aéreo desses aeroportos. Nas Conclusões são destacados alguns cuidados a serem observados na busca de ampliação da capacidade de um sistema aeroportuário.

\section{Capacidade do subsistema pistas.}

$\mathrm{Na}$ análise da literatura podem ser identificadas duas metodologias para quantificação da capacidade do subsistema pistas. Uma desenvolvida para a Federal Aviation Administration (FAA, 1999), órgão norte-americano subordinado ao USDoT (Departamento de Transportes) onde se parte de uma série de hipóteses e considerações para se quantificar, com o emprego de ábacos, o movimento de aeronaves e os atrasos médios por operação. Outra, adotada pelo Departamento de Controle do Espaço Aéreo (DECEA), organização militar do Comando da Aeronáutica, responsável pelo planejamento e gerenciamento das atividades relacionadas ao controle do espaço aéreo no Brasil, que estipula quantitativos de movimentos com o uso de técnicas de simulação, tendo como base as condições locais e as separações praticadas no País (CGNA, 2011). 
Alguns fatores intervenientes são computados em uma ou outra metodologia de forma diferente. Os dados de entrada para o método FAA são basicamente: configuração do sistema de pistas, número de saídas, composição da frota operante (mix), porcentagem de pousos na hora-pico, posição das saídas de pista, quantidade de manobras de toque-e-arremetidas ${ }^{2}$ e tipo de operação (VFR ou IFR). Para o método DECEA além desses dados, ganham importância as separações entre as operações, determinadas pelas características locais e pelas regras de tráfego aéreo (DECEA, 2006). Esse número estipulado, que serve como referência para a autorização de voos, corresponde a $80 \%$ do máximo teórico. Essa porcentagem de $20 \%$ dá margem a acomodação de contingências ou situações inesperadas.

Na Tabela 1 associa-se os 20 aeroportos em estudo (sigla IATA) aos valores de capacidade de pista estabelecidos pelo Centro de Gerenciamento de Navegação Aérea, CGNA (DECEA1 dados de 2009 e DECEA2 - dados de 2011) e pelo método FAA (McKinsey \& Company, 2010), sequenciado pela movimentação de passageiros em 2010 (INFRAERO, 2011).

Tabela 1 - Capacidade do subsistema pistas (operações por hora)

\begin{tabular}{|c|c|c|c|c|c|c|}
\hline Ranking & Cidade & Aeroporto & Movimento & DECEA1 & DECEA2 & FAA \\
\hline 1 & São Paulo & GRU & 26.774 .546 & 49 & 45 & 58 \\
\hline 2 & São Paulo & CGH & 15.481 .370 & 34 & 34 & 50 \\
\hline 3 & Brasília & BSB & 14.149 .306 & 50 & 45 & 46 \\
\hline 4 & Rio & GIG & 12.229 .513 & 48 & 40 & 58 \\
\hline 5 & Rio & SDU & 7.805 .387 & 33 & 29 & 40 \\
\hline 6 & Salvador & SSA & 7.540 .298 & 32 & 25 & 50 \\
\hline 7 & BH & CNF & 7.261 .041 & 32 & 27 & 48 \\
\hline 8 & Porto Alegre & POA & 6.676 .216 & 28 & 25 & 48 \\
\hline 9 & Recife & REC & 5.933 .137 & 28 & 29 & 50 \\
\hline 10 & Curitiba & CWB & 5.769 .712 & 38 & 24 & 46 \\
\hline 11 & Fortaleza & FOR & 5.072 .786 & 28 & 27 & 46 \\
\hline 12 & Campinas & VCP & 5.021 .939 & 31 & 26 & 46 \\
\hline 13 & Manaus & MAO & 2.705 .131 & 28 & nd & 44 \\
\hline 14 & Florianópolis & FLN & 2.672 .250 & 33 & 15 & 42 \\
\hline 15 & Vitória & VIX & 2.644 .729 & 26 & 11 & 48 \\
\hline 16 & Belém & BEL & 2.570 .890 & 34 & 20 & 48 \\
\hline 17 & Natal & NAT & 2.413 .416 & 36 & 27 & 55 \\
\hline 18 & Goiânia & GYN & 2.348 .648 & 28 & 26 & 49 \\
\hline 19 & Cuiabá & CGB & 2.134 .267 & 26 & 22 & 49 \\
\hline 20 & BH & PLU & 757.685 & 28 & 25 & 48 \\
\hline
\end{tabular}

nd - não declarada

\footnotetext{
${ }^{2}$ Operação que consiste em uma aeronave tocar e decolar de uma pista de pouso, sem parar sobre a mesma ou livrá-la (DECEA, 2006).
} 
A última coluna, os resultados obtidos pelo método FAA, mostra valores bem mais elevados. Isso pode ser atribuído a não aderência da realidade brasileira às condições operacionais consideradas pela FAA. De fato, a separação entre aeronaves mantida no Brasil é mais do que o dobro da prevista pelo órgão norteamericano. Observa-se também, de forma geral, uma diminuição dos valores adotados pelo CGNA de 2009 para os divulgados em 2011 (excetuando São Paulo/Congonhas e Recife que se manteve ou teve ligeiro aumento). A redução mais significativa está localizada em aeroportos dotados de mais de uma pista de pouso e aonde acontecem cruzamentos de trajetórias: Curitiba, Florianópolis, Belém e Natal. Não foi possível identificar o motivo para a redução expressiva dos voos autorizados em Vitória. Vale frisar que os valores estipulados pelo DECEA representam, segundo informações daquela organização, $80 \%$ da capacidade teórica máxima.

Em função da consideração das restrições vigentes, neste trabalho a capacidade considerada do subsistema pista é a estipulada pelo DECEA em 2011 (coluna DECEA2 da Tabela 1).

Essa capacidade pode ser ampliada, mesmo sem a realização de obras, através de um melhor gerenciamento das operações, que viabilize separações menores entre aeronaves. $\mathrm{O}$ desenho de novas trajetórias de descida e subida considerando o apoio dos sinais satelitais e o treinamento dos recursos humanos que atuem no controle desses tráfegos são imprescindíveis.

Medau (2011) com o uso de simulação (Visual SIMMOD) analisa a variação da capacidade de pistas com a modificação de procedimentos utilizados pelos controladores de tráfego aéreo, respeitando-se critérios de nível de serviço e recomendações da ICAO. Fraga (2010/2011) analisa a variação da capacidade de pistas com a implantação de novos procedimentos de aproximação, assim como a restrição de capacidade imposta pela sopreposição de procedimentos para diferentes aeroportos dentro de uma mesma área de controle terminal. 


\section{Capacidade do subsistema pátios.}

Já a avaliação da capacidade de pátios de estacionamento de aeronaves depende de:

- Posições de estacionamento - quantidade, tamanho, restrições de uso (compartilhado ou exclusivo), tipo de operação (doméstica ou internacional), localização (próxima ou remota) e disposição geométrica em relação ao terminal;

- Tempo de permanência nas posições;

- Frota de aeronaves em operação - porte das mesmas e tipo de operação; e,

- Porcentagem de voos domésticos e internacionais.

O fluxograma traçado na Figura 1 revela a metodologia utilizada para a quantificação da capacidade dos pátios de estacionamento dos aeroportos brasileiros.

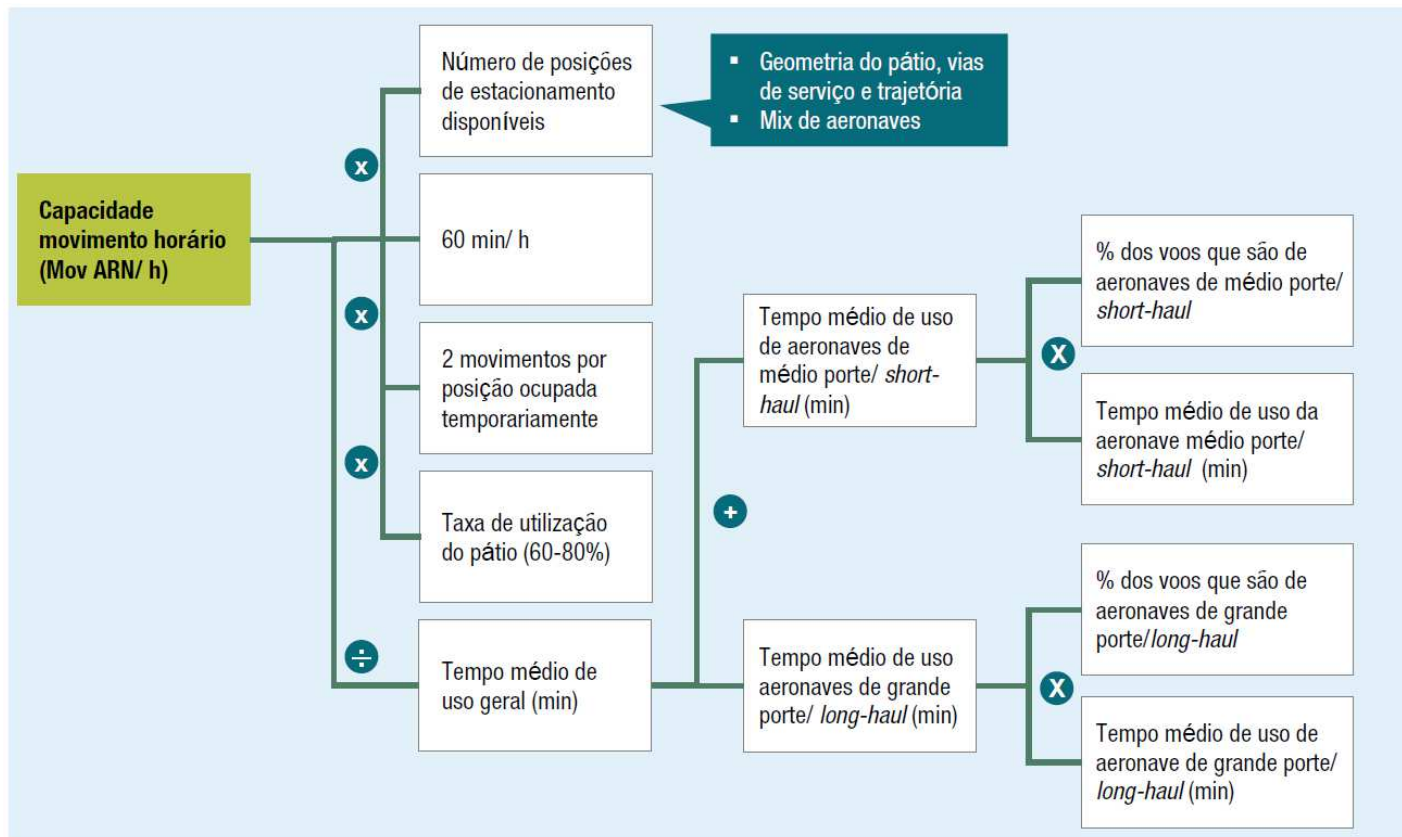

Figura 1 - Fluxograma para avaliação da capacidade de pátios (Fonte: McKinsey \& Company, 2010) 
Na Tabela 2 estão os resultados obtidos em termos de movimento de aeronaves por hora, considerando-se a frota operante em cada aeroporto na hora-pico de dezembro de 2009 . Na última coluna registra-se o valor teórico dessa capacidade se forem reduzidos os tempos médios de permanência em $20 \%$ e, conjuntamente, se a taxa de utilização do pátio for elevada em $10 \%$. Os dados utilizados para cada aeroporto foram os mesmos obtidos para o estudo da McKinsey (2010), referentes, portanto, ao ano de 2009.

Possíveis formas de ampliar a capacidade do pátio de estacionamento de aeronaves, sem expandir sua área implantada, estão na possibilidade em se alterar os parâmetros intervenientes., como: redução do tempo médio de permanência das aeronaves na posição (por exemplo, através de tarifa diferenciada); redução das separações de segurança entre as aeronaves estacionadas (desde que se estabeleça um monitoramento ou coordenação que garanta a segurança das operações na área de rampa); e viabilizar uma política de uso compartilhado de equipamentos de solo, diminuindo a circulação de veículos e equipamentos na área do pátio. Várias dessas iniciativas confrontam-se com a prática operacional empregada nos aeroportos brasileiros e na dificuldade para uma mudança cultural dentro das empresas envolvidas, onde a competição supera a cooperação pelo uso mais eficiente dos recursos disponíveis. 
Tabela 2 - Capacidade do subsistema pátios (movimento de aeronaves por hora)

\begin{tabular}{|c|c|c|c|c|c|}
\hline Ranking & Cidade & Aeroporto & $\begin{array}{c}\text { Capacidade } \\
\text { Pistas }\end{array}$ & $\begin{array}{c}\text { Capacidade } \\
\text { Pátios } 1\end{array}$ & $\begin{array}{c}\text { Capacidade } \\
\text { Pátios2 }\end{array}$ \\
\hline 1 & São Paulo & GRU & 45 & 35,6 & 49,0 \\
\hline 2 & São Paulo & CGH & 34 & 36,3 & 49,9 \\
\hline 3 & Brasília & BSB & 45 & 36,8 & 50,6 \\
\hline 4 & Rio & GIG & 40 & 47,9 & 65,8 \\
\hline 5 & Rio & SDU & 29 & 27,6 & 38,0 \\
\hline 6 & Salvador & SSA & 25 & 15,2 & 20,8 \\
\hline 7 & BH & CNF & 27 & 23,5 & 32,3 \\
\hline 8 & Porto Alegre & POA & 25 & 23,7 & 32,6 \\
\hline 9 & Recife & REC & 29 & 25,1 & 34,6 \\
\hline 10 & Curitiba & CWB & 24 & 25,1 & 34,5 \\
\hline 11 & Fortaleza & FOR & 27 & 15,8 & 21,7 \\
\hline 12 & Campinas & VCP & 26 & 14,7 & 20,3 \\
\hline 13 & Manaus & MAO & nd & 11,8 & 16,2 \\
\hline 14 & Florianópolis & FLN & 15 & 10,4 & 14,3 \\
\hline 15 & Vitória & VIX & 11 & 7,4 & 10,2 \\
\hline 16 & Belém & BEL & 20 & 11,9 & 16,4 \\
\hline 17 & Natal & NAT & 27 & 8,3 & 11,4 \\
\hline 18 & Goiânia & GYN & 26 & 8,7 & 11,9 \\
\hline 19 & Cuiabá & CGB & 22 & 10,6 & 14,5 \\
\hline 20 & BH & PLU & 25 & 12,2 & 16,8 \\
\hline
\end{tabular}

nd - não declarada

Em função do exposto, nesse trabalho, a capacidade do pátio a ser considerada é a mostrada na quinta coluna da Tabela 2 (DECEA1), isto é, não considerando a hipótese de alterações no modus operandi atual.

\section{Capacidade do lado aéreo do aeroporto.}

Baseada nas duas tabelas anteriores foi traçada a Figura 2 onde se associa o gargalo operacional do lado aéreo com os aeroportos estudados. A capacidade do lado aéreo dos aeroportos de São Paulo (CGH), Rio (GIG) e Curitiba (CWB) apresenta como limitante a cadência operacional do subsistema pistas. Significa que ganhos implementados nesse subsistema ampliam efetivamente a capacidade do lado aéreo desses aeroportos. 


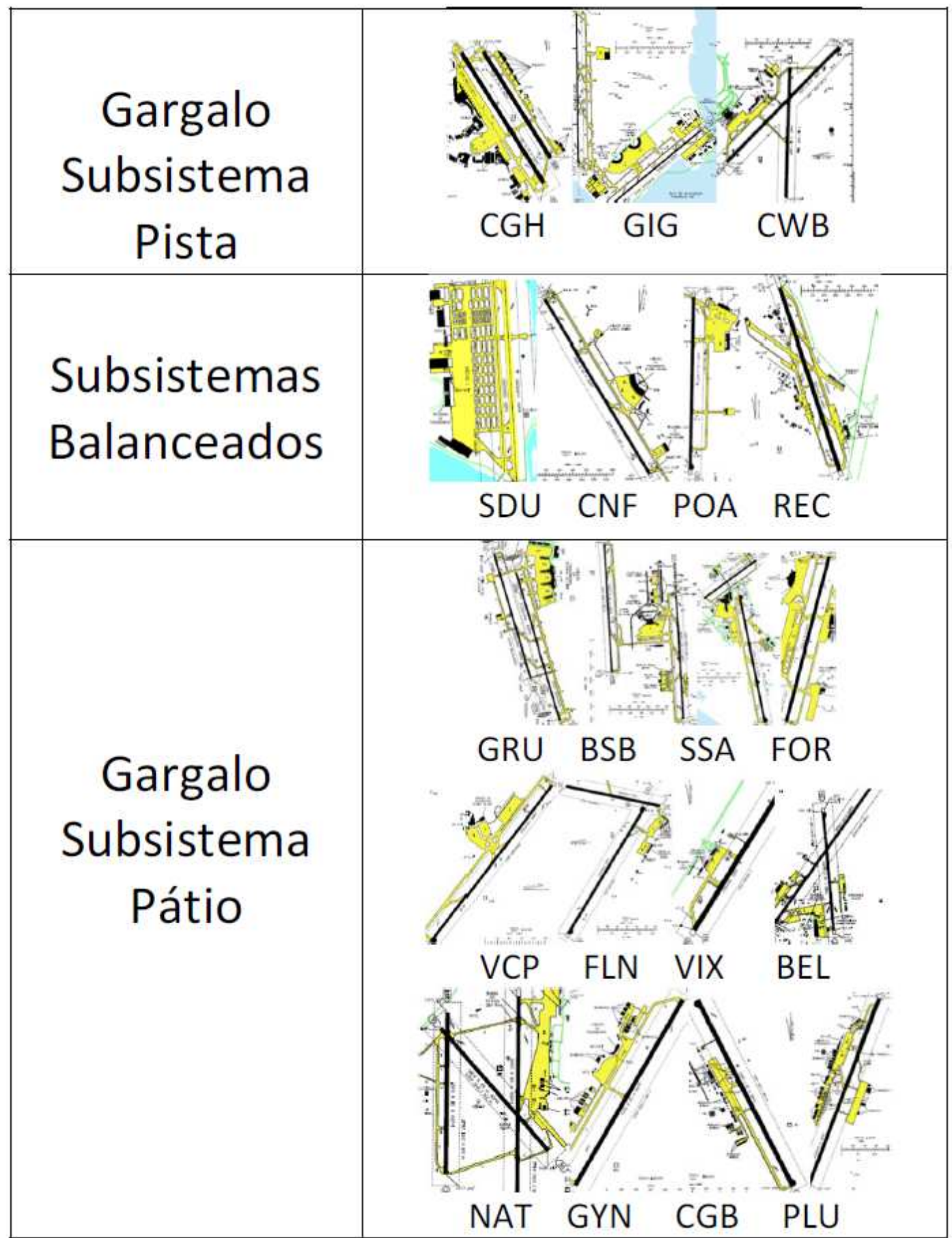

Figura 2 - Gargalos do lado aéreo nos aeroportos estudados

Já os aeroportos de Rio (SDU), Belo Horizonte (CNF), Porto Alegre (POA) e Recife (REC) mostram um equilíbrio entre os dois subsistemas do lado aéreo, ganhos que se implementem em um deles, automaticamente atribui ao outro subsistema a condição de limitante (gargalo).

Na maioria dos aeroportos estudados: São Paulo (GRU), Brasília (BSB), Salvador (SSA), Fortaleza (FOR), Campinas (VCP), Florianópolis (FLN), Vitória (VIX), Belém (BEL), Natal 
(NAT), Goiânia (GYN), Cuiabá (CGB) e Belo Horizonte (PLU) a limitação da operação do lado aéreo se verifica no subsistema pátios. Isto é, somente justificam ampliações nas pistas se houver expansões de maior vulto no subsistema pátio.

\section{Conclusões}

Algumas conclusões podem ser enumeradas quanto à abordagem desse estudo. Primeiramente, não se justificam expansões em capacidade de um subsistema se não houver a verificação do balanceamento entre as capacidades dos demais elementos do sistema. Pois nesses casos não se estaria ampliando a capacidade do aeroporto, apenas daquele subsistema, e se continuaria limitado pelo subsistema gargalo.

Dos 20 maiores aeroportos brasileiros em número de passageiros transportados, apenas 4 mostram um balanceamento de capacidades dentro do lado aéreo. Isso não significa que a capacidade instalada nos mesmos seja suficiente diante da pressão da demanda. O que mostra é que em SDU, CNF, POA e REC os subsistemas pistas e pátios estão com capacidade instalada equilibrada. $\mathrm{O}$ aumento da capacidade desses aeroportos em seu lado aéreo necessita de ações em ambos os subsistemas.

Excetuando SDU, CNF, POA, REC, CGH, GIG e CWB, seria recomendável, para os demais 13 aeroportos, que fossem promovidas ações no sentido de ampliar a capacidade dos pátios de estacionamento de aeronaves, para se conseguir expandir efetivamente a capacidade do lado aéreo desses aeroportos.

Enfatiza-se que, apesar de nesse artigo se restringir a apreciação da capacidade do lado aéreo dos aeroportos, qualquer ampliação de um subsistema aeroportuário deve ser realizada com a análise das capacidades dos demais componentes do sistema (subsistemas). Constata-se que muitas obras, ainda que propiciem maiores níveis de segurança às operações, não geram o aumento de capacidade que o aeroporto precisa por, na realidade, estarem transferindo o gargalo do aeroporto para outro subsistema limitante. 


\section{Referências}

Carvalho, B. G. (2006) Uma metodologia para obtenção de um diagnóstico dos principais aeroportos no Brasil através da relação demanda e capacidade. Tese de Mestrado, Instituto Tecnológico de Aeronáutica - ITA. São José dos Campos.

CGNA (2011) Centro de Gerenciamento da Navegação Aérea. Fonte: http://www.cgna.gov.br/

DECEA (2006) ICA 100-12. Departamento de Controle do Espaço Aéreo. Rio de Janeiro.

DECEA (2006) CIRTRAF 100-3. Departamento de Controle do Espaço Aéreo. Rio de Janeiro.

FAA (1999) Federal Aviation Administration. AC 150/5060-5. Washington.

Fraga, R.; Müller, C.; Alves, C. J. P. (2010) Analysis of criteria for reduced-spaced parallel runway approaches: the application of PRM/SOIA in Sao Paulo Airport. - 12th WCTR, Lisboa.

Fraga, R.; Müller, C.; Alves, C. J. P. (2011) Analysis of Criteria for Closely-Spaced Parallel Runway Approaches: Influences and Capacity Constraints in a Multiple Airport System. T\&DI Congress 2011, Chicago.

FSF (2009) Flight Safety Foundation - ALAR Briefing Note 8.1 - Runway Excursions and Runway Overruns. Fonte: http://www.skybrary.aero/bookshelf/books/865.pdf

Gualda, N. D. (1995) Terminais de transportes: contribuição ao planejamento e ao dimensionamento operacional. Livre Docência. EPUSP. São Paulo.

INFRAERO (2011) Empresa Brasileira de Infra-Estrutura Aeroportuaria. Fonte: http://www.infraero.gov.br

McKinsey \& Company (2010) Estudo do setor do transporte aéreo do Brasil. Rio de Janeiro.

Medau, J. (2011) Análise de capacidade do lado aéreo de aeroportos baseada em simulação computacional: aplicação ao Aeroporto de São Paulo - Congonhas. Mestrado. EPUSP. São Paulo. 